\title{
THE INITIAL VALUE PROBLEM FOR NONLINEAR WAVE EQUATIONS IN HILBERT SPACE $\left({ }^{1}\right)$
}

\author{
BY \\ LUIZ ADAUTO MEDEIROS
}

1. Introduction. The primary purpose of this paper is to generalize Browder's results contained in [1], to the case of wave equations with time variable coefficients. The origin of this problem may be presented as follows. In the applications of mathematical methods to physics, one is often concerned with the study of nonlinear partial differential equations, for example, the Klein-Gordon equation

$$
\partial^{2} u / \partial t^{2}-\Delta u+\mu^{2} u+\eta^{2} u^{3}=0,
$$

where $\mu, \eta$ are constants and $\Delta$ is the Laplace operator.

K. Jörgens in Math. Z. 77 (1961), 295-308, has studied the Cauchy initial value problem for equations of the form

$$
\partial^{2} u / \partial t^{2}-\Delta u+F^{\prime}\left(|u|^{2}\right) u=0
$$

which are generalizations of (1), where $F$ is a numerical function with derivative $F^{\prime}$. Jörgens used concrete analytical methods in order to obtain his results.

Browder in [1], has given the proof of the solvability of the Cauchy initial value problem for a class of operator differential equations in an abstract Hilbert space, which contains the equation (2) as a particular case. Following is a summary of Browder's ideas contained in [1]. Given a Hilbert space $H$, let $A$ be a selfadjoint and positive semibounded operator with domain $D(A)$, dense in $H$, and range in $H$ and $M(u)$ be a nonnecessarily linear mapping from $D\left(A^{1 / 2}\right)$ into $H$ satisfying certain conditions (cf. [1, conditions I, II, III, IV]). Let us consider the vector functions $u: \boldsymbol{R} \rightarrow D(A)$, where $\boldsymbol{R}$ is the field of real numbers, twice strongly continuously differentiable in $H$ and the operator differential equations:

$$
d^{2} u / d t^{2}+A u+M(u)=0 .
$$

Browder has given the proof of the solvability of the Cauchy initial value problem for the strict solutions of (3), when the initial data $\left(u(0), u^{\prime}(0)\right)$ is prescribed in $D(A) \times D\left(A^{1 / 2}\right)$. He used the operational calculus for selfadjoint operators in Hilbert spaces and the Picard method of successive approximations. An application is given when $A$ is a selfadjoint realization of an elliptic partial differential operator

Received by the editors May 18, 1966 and, in revised form, November 9, 1967.

( $\left.{ }^{1}\right)$ This research was supported by National Science Foundation Grant 19751, Yale University and Conselho Nacional de Pesquisas (Brasil). Ph.D. dissertation, presented to the Faculty of the Institute for Pure and Applied Mathematics, Rio de Janeiro, Brasil. 
on the Hilbert space $L^{2}\left(R^{N}\right)$ and $M(u)=F^{\prime}\left(|u|^{2}\right) u$ with convenient hypothesis on $F$, and then he obtained Jörgens results.

The equations (3) are taken as the model for our study. The unique novelty, in this step of our research, is the perturbation that shall be introduced in the coefficients of (3), i.e., we shall consider equations of the form

$$
d^{2} u / d t^{2}+A(t) u+M(u)=0,
$$

where $A(t)$ is a family of operators with domain and range in $H$ and for technical reasons, we shall assume $M(u)$ is a map from $D\left(A(0)^{1 / 2}\right)$ into $D\left(A(0)^{1 / 2}\right)$. The method used by Browder, cannot be adapted naturally to the case of equations (4). Instead, by a convenient change of variable, the Cauchy problem for (4) shall be transformed in a nonlinear first order system (cf. $\$ 5,(20)$ and (21)), and the latter system can be considered as a nonlinear first order equation (cf. $\$ 5$ (24) and (25)), in the Hilbert space $\mathscr{H}$, which is the direct sum of $H$ with $H$. To solve the nonlinear Cauchy problem, we have used the Kato's results, (cf. [6]) and the Picard-Banach fixed point theorem.

In $\$ 5$ we shall give an example of a family of partial differential operators, realized on $L^{2}(G)$, where $G$ is a bounded open set in $R^{N}$, which satisfies the abstract hypothesis assumed on the coefficient $A(t)$ of the equation (4).

I take this opportunity, to express my appreciation to Professor Felix E. Browder, who proposed this question to me, for his assistance and valuable suggestions during the period of research on the problem.

To Professor Leopoldo Nachbin, I express my personal gratitude for his constant assistance and encouragement, particularly when I was research assistant at the Institute for Pure and Applied Mathematics (Rio de Janeiro, Brasil), during the years 1959-1961.

I would also like to express my appreciation to Professor Frank J. Hahn, for his many discussions with me on the subject, when I was at Yale University during the years 1962-1964.

2. On the domain of the square root. We denote by $H$ a Hilbert space with inner product $(\mid)$ and norm \|\| . Let $\{A(t), t \geqq 0\}$ be a family of selfadjoint operators of $H$, bounded below by a bounded continuous function $k(t)>0$, i.e., $(A(t) u \mid u) \geqq k(t)(u \mid u)$ for all $u$ in the domain of $A(t)$, which shall be represented by $D(A(t))$. If $A=A\left(t_{0}\right)$ is an operator in that family, then we consider the positive square root of $A$, represented by $A^{1 / 2}$. As a consequence of the spectral decomposition, it follows that $D(A) \subseteq D\left(A^{1 / 2}\right)$.

In the following, we will construct $D\left(A^{1 / 2}\right)$ from $D(A)$ by a method used by Friedrichs to obtain selfadjoint extensions for semibounded symmetric operators (cf. Riesz-Sz. Nagy, Functional analysis, Ungar, New York, 1955, §124, p. 331). By hypotheses, we have $(A u \mid u) \geqq k(u \mid u)$, and let us suppose that $k=1$. If we define in $D(A)$ a new inner product by $[u \mid v]=(A u \mid v)$, it follows that the norm induced in $D(A)$ by [ | ], is greater than the original norm of $H$, i.e., $[u \mid u]=(A u \mid u) \geqq(u \mid u)$. 
Let us represent by [[ ]] the norm induced by [ | ]. $D(A)$ equipped with [[ ]] is a pre-Hilbert space. The completion of $D(A)$ with respect to [[ ]], can be identified with a subspace $H_{0}$ of $H$, and we have $D(A) \subseteq H_{0} \subseteq H$. We shall prove in Proposition 1 , that $H_{0}=D\left(A^{1 / 2}\right)$. We say that a sequence $\left\{u_{n}\right\} \subset D(A)$ converges to $u \in D\left(A^{1 / 2}\right)$ in the graph norm, if and only if $\left\|u_{n}-u\right\| \rightarrow 0$ and $\left\|A^{1 / 2} u_{n}-A^{1 / 2} u\right\| \rightarrow 0$.

LEMma 1. The domain of $A$ is dense in the domain of $A^{1 / 2}$ with respect to the graph norm.

Proof. In fact, let $E(\lambda),-\infty<\lambda<+\infty$, be the spectral family associated to $A$. Since $A \geqq 1$, we have $E(\lambda)=0$ for $\lambda<1$. For $v \in D\left(A^{1 / 2}\right), v_{n}=E(n) v$, for each positive integer $n$ belongs to $D(A)$ and $\lim v_{n}=v, \lim A^{1 / 2} v_{n}=A^{1 / 2} v$ with respect to the norm of $H$.

Proposition 1. The completion $H_{0}$ of $D(A)$ is the domain of the square root $A^{1 / 2}$.

Proof. If $u \in H_{0}$, there exists a sequence $\left\{u_{n}\right\}, u_{n} \in D(A)$, such that $\left\{u_{n}\right\}$ is a Cauchy sequence in $H_{0}$ normed by [[ ]], and converges to $u$ in the original metric of $H$. It follows that $\left\{A^{1 / 2} u_{n}\right\}$ is a Cauchy sequence of $H$, and thus convergent. Hence it follows that $u \in D\left(A^{1 / 2}\right)$, since $A^{1 / 2}$ is closed.

Let $u$ be a vector of $D\left(A^{1 / 2}\right)$. By Lemma 1 , there exists a sequence $\left\{u_{n}\right\} \subset D(A)$ such that $u_{n} \rightarrow u$ and $A^{1 / 2} u_{n} \rightarrow A^{1 / 2} u$ in $H$. Thereby $\left\{u_{n}\right\}$ is a Cauchy sequence in the metric given by [[ ]] and it follows that $u \in H_{0}$.

Proposition 2. Let $A_{1}=A\left(t_{1}\right), A_{2}=A\left(t_{2}\right)$ be two operators of the family $\{A(t), t \geqq 0\}$ such that $D\left(A_{1}\right) \subseteq D\left(A_{2}\right)$. If there exists a constant $c>0$ such that $\left(A_{2} u \mid u\right) \leqq c\left(A_{1} u \mid u\right)$ for all $u$ in $D\left(A_{1}\right)$, then $D\left(A_{1}^{1 / 2}\right) \subseteq D\left(A_{2}^{1 / 2}\right)$.

Proof. In fact, let $u$ be an element of $D\left(A_{1}^{1 / 2}\right)$. Then, there exists a sequence $\left\{u_{n}\right\}$ contained in $D\left(A_{1}\right)$ such that $u_{n} \rightarrow u$ and $A_{1}^{1 / 2} u_{n} \rightarrow A_{1}^{1 / 2} u$. It follows that $\left\{u_{n}\right\}$ is a Cauchy sequence with respect to the metric given by $[[v]]_{2}=\left(A_{2} v \mid v\right)$, since $\left(A_{2} v \mid v\right) \leqq c\left(A_{1} v \mid v\right)$. Hence, $u_{n} \rightarrow u$ and $A_{2}^{1 / 2} u_{n} \rightarrow w$ in $H$. Therefore $u \in D\left(A_{2}^{1 / 2}\right)$.

REMARK 1. The result contained in the Proposition 2, is a natural consequence of the method used to construct the domain of the square root. In fact, if we define $[[u]]_{1}=\left(A_{1} u \mid u\right)$ and $[[u]]_{2}=\left(A_{2} u \mid u\right)$, we have by hypotheses $[[u]]_{2} \leqq c[[u]]_{1}$, for all $u$ in $D\left(A_{1}\right)$. Hence, it follows that there are at least as many Cauchy sequences with respect to $[[]]_{2}$ as with respect to $[[]]_{1}$.

Remark 2. Assuming the hypotheses of the Proposition 2, if $D\left(A_{2}\right) \subseteq D\left(A_{1}\right)$ and if there exists a constant $c^{\prime}>0$ such that $\left(A_{1} u \mid u\right) \leqq c^{\prime}\left(A_{2} u \mid u\right)$ for all $u$ in $D\left(A_{2}^{1 / 2}\right)$, it follows that $D\left(A_{2}^{1 / 2}\right) \subseteq D\left(A_{1}^{1 / 2}\right)$.

REMARK 3. When $D\left(A_{1}\right)=D\left(A_{2}\right)$ and there are constants $c>0, c^{\prime}>0$ such that

$$
c^{\prime}\left(A_{1} u \mid u\right) \leqq\left(A_{2} u \mid u\right) \leqq c\left(A_{1} u \mid u\right)
$$

for all $u$ in $D\left(A_{1}\right)=D\left(A_{2}\right)$, then $D\left(A_{1}^{1 / 2}\right)=D\left(A_{2}^{1 / 2}\right)$. 
LEMMA 2. If $A$ is an operator of the family $\{A(t), t \geqq 0\}$, its range is equal to the space $H$.

Proof. The range of $A$ is dense in $H$ because $A$ is selfadjoint. It is sufficient to prove that, if $u \in H$ then $u$ belongs to the range of $A$. In fact, if $u \in H$, there exists a sequence $\left\{u_{n}\right\}$ contained in the range of $A$ such that $u_{n} \rightarrow u$ in $H$. Let $v_{n} \in D(A)$ be such that $A v_{n}=u_{n}, n=1,2, \ldots$ Hence it follows that

$$
0<k\left\|v_{n}-v_{m}\right\|^{2} \leqq\left(A v_{n}-A v_{m} \mid v_{n}-v_{m}\right) \leqq\left\|A v_{n}-A v_{m}\right\| \cdot\left\|v_{n}-v_{m}\right\| .
$$

Whence, we have that $\left\{v_{n}\right\}$ is a Cauchy sequence in $H$, then convergent to a vector $v \in D(A)$ and such that $A v=u$.

By hypotheses on the family $\{A(t)\}$, we know that $0<k(t)(u \mid u) \leqq(A(t) u \mid u)$ $\leqq\|A(t) u\| \cdot\|u\|$, for all nonzero $u$ in $D(A(t))$. Then, it follows that the inverse $A(t)^{-1}$ exists for each $t \geqq 0$, and by Lemma 2 , its domain is the space $H$. We need to use the product $A(t) \cdot A(s)^{-1}$ for $t \neq s$. Such a product is always defined when $D(A(t))$ is constant with respect to $t$. In order to avoid difficulties, we shall make the following assumption on $A(t)$.

Assumption I. The domain $D(A(t))$ is constant for $t \geqq 0$ i.e.

$$
D(A(t))=D(A(s)) \text { for all } t, s \geqq 0 .
$$

By Assumption I, it follows that the operators $A(s) \cdot A(t)^{-1}$ are well defined for all $s, t \geqq 0$ and are bounded, by the closed graph theorem.

Assumption II. The bounded operators $A(t) \cdot A(s)^{-1}$ are uniformly bounded and satisfy Lipschitz conditions with respect to $t$, i.e., there exist positive constants $M$ and $k$, such that $\left\|A(t) A(s)^{-1}\right\| \leqq M$ and $\left\|A(t) A\left(t_{0}\right)^{-1}-A(s) A\left(t_{0}\right)^{-1}\right\| \leqq k|t-s|$ for all $t, t_{0}$ and $s$.

REMARK 6. By Remark 3, we conclude that when $D(A(t))=D(A(0))=D$ and if there exist continuous positive real functions $c(t), c^{\prime}(t)$ on the semiline $t \geqq 0$ such that

$$
c^{\prime}(t)(A(0) u \mid u) \leqq(A(t) u \mid u) \leqq c(t)(A(0) u \mid u)
$$

for all $u$ in $D$, then $D\left(A(t)^{1 / 2}\right)=D\left(A(0)^{1 / 2}\right)$ for all $t$.

We will prove, in the next theorem, that the Assumptions I and II are sufficient conditions for the independence of $D\left(A(t)^{1 / 2}\right)$ with respect to $t>0$.

THEOREM 1. If $\{A(t), t \geqq 0\}$ is a family of operators satisfying the Assumptions I and II, then $D\left(A(t)^{1 / 2}\right)$ is independent of $t$ for $t \geqq 0$.

Proof. In fact, the operators $A(t) A(s)^{-1}-A(0) A(s)^{-1}$ are bounded.

Hence, it follows that for each $u$ in $D(A(t))=D$, we have

$$
\begin{aligned}
\|A(t) u-A(0) u\| & =\left\|\left[A(t) A(s)^{-1}-A(0) A(s)^{-1}\right]\left[A(s) A(0)^{-1}\right] A(0) u\right\| \\
& \leqq\left\|A(t) A(s)^{-1}-A(0) A(s)^{-1}\right\| \cdot\left\|A(s) A(0)^{-1}\right\| \cdot\|A(0) u\| \\
& \leqq M k t\|A(0) u\| .
\end{aligned}
$$


Whence, it follows

$$
\|A(t) u\| \leqq\|A(t) u-A(0) u\|+\|A(0) u\| \leqq c(t)\|A(0) u\|
$$

for all $u$ in $D$, where $c(t)=M k t+1$ is always positive for $t>0$. By Satz 3 of Heinz [5], (also Kato [7, Theorem 2]), we have

$$
\left\|A(t)^{1 / 2} u\right\| \leqq k(t)\left\|A(0)^{1 / 2} u\right\|
$$

for all $u \in D$, where $k(t)=+c(t)^{1 / 2}$.

Since we also have

$$
\|A(t) u-A(0) u\| \leqq\left\|\left[A(t) A(s)^{-1}-A(0) A(s)^{-1}\right]\left[A(s) A(t)^{-1}\right] A(t) u\right\|,
$$

we obtain by the same argument

$$
\left\|A(0)^{1 / 2} u\right\| \leqq k(t)\left\|A(t)^{1 / 2} u\right\| .
$$

By (5) and (6) we have

$$
c(t)^{-1}\left\|A(0)^{1 / 2} u\right\|^{2} \leqq\left\|A(t)^{1 / 2} u\right\|^{2} \leqq c(t)\left\|A(0)^{1 / 2} u\right\|^{2}
$$

for all $u$ in $D$. Whence it follows that $D\left(A(t)^{1 / 2}\right)$ is independent of $t$ (cf. Remark 6).

3. Integral representation of $A^{\alpha}$. In this paragraph, we will prove an integral representation for the fractional power $A^{\alpha}, 0<\alpha<1$, of a positive selfadjoint operator $A$. Let $f(\lambda)=\lambda^{\alpha}$ be the positive real branch of the mapping $\lambda \rightarrow \lambda^{\alpha}$ of $\boldsymbol{R}$ into $\boldsymbol{R}$. By the spectral decomposition, we have

for all $u$ in $D\left(A^{\alpha}\right)$.

$$
A^{\alpha} u=\int_{0}^{\infty} \lambda^{\alpha} d E(\lambda) u
$$

Our next step will be to obtain, from (7), another integral representation of $A^{\alpha}$. First of all, we observe that for $t>0$ we know that $(A \pm i t)^{-1}, i^{2}=-1$, exists, is defined and bounded on $H$.

Let us prove that for $0<\alpha<1$, the mapping

$$
t \rightarrow t^{\alpha}\left\{(A+i t)^{-1}+(A-i t)^{-1}\right\} u
$$

of $0 \leqq t<+\infty$ into $H$ is integrable on $0 \leqq t<\infty$ for each $u \in D\left(A^{\alpha}\right)$ and we have

$$
A^{\alpha} u=\frac{\cos (\alpha \pi / 2)}{\pi} \int_{0}^{\infty} t^{\alpha}\left\{(A+i I)^{-1}+(A-i I)^{-1}\right\} u d t
$$

In fact, we have

$$
\left\{(A+i t)^{-1}+(A-i t)^{-1}\right\} u=\int_{0}^{\infty} \frac{2 \lambda}{\lambda^{2}+t^{2}} d E(\lambda) u
$$

for all $u$ in $H$.

Suppose $0<t_{1}<t_{2}<+\infty$ and taking the product of both sides of (9) with $t^{\alpha}$ and integrating from $t_{1}$ to $t_{2}$, we obtain

$$
\int_{t_{1}}^{t_{2}} t^{\alpha}\left\{(A+i t)^{-1}+(A-i t)^{-1}\right\} u d t=\int_{0}^{\infty}\left\{\int_{t_{1}}^{t_{2}} \frac{2 t^{\alpha} \lambda}{\lambda^{2}+t^{2}} d t\right\} d E(\lambda) u
$$


For all $u$ in $D\left(A^{\alpha}\right)$, we obtain

$$
\begin{gathered}
\left\|A^{\alpha} u-\frac{\cos (\pi \alpha / 2)}{\pi} \int_{\mathrm{t}_{1}}^{t_{2}} t^{\alpha}\left\{(A+i t)^{-1}+(A-i t)^{-1}\right\} u d t\right\| \\
=\left\|\int_{0}^{\infty} \lambda^{\alpha}\left(1-\frac{2 \cos (\alpha \pi / 2)}{\pi} \int_{t_{1} / \lambda}^{t_{2} / \lambda} \frac{s^{\alpha} d s}{1+s^{2}}\right) d E(\lambda) u\right\|^{2} \\
=\int_{0}^{\infty}\left(1-\frac{2 \cos (\alpha \pi / 2)}{\pi} \int_{t_{1} / \lambda}^{t_{2} / \lambda} \frac{s^{\alpha} d s}{1+s^{2}}\right)^{2} \lambda^{2 \alpha} d(E(\lambda) u \mid u) .
\end{gathered}
$$

Observe that $\lambda^{2 \alpha}$ is integrable with respect to the measure $\sigma(\lambda)=(E(\lambda) u \mid u)$, because $u \in D\left(A^{\alpha}\right)$ by hypotheses. Furthermore, we have

$$
\int_{0}^{\infty} \frac{s^{\alpha} d s}{1+s^{2}}=\frac{\pi}{2 \cos (a \pi / 2)}, \quad 0<\alpha<1
$$

Making $t_{1} \rightarrow 0$ and $t_{2} \rightarrow \infty$ in (10), we obtain the representation (8).

If we decompose the integral (8) in two pieces and change the variables in the second integral, we obtain

$$
A^{\alpha} u=\frac{\cos (\alpha \pi / 2)}{\pi} \int_{-\infty}^{+\infty}|t|^{\alpha}(A+i t)^{-1} u d t
$$

for all $u$ in $D\left(A^{\alpha}\right)$.

When we have a family of selfadjoint operators as in $\S 2$, then by (11) we have

$$
A(t)^{\alpha} u=\frac{\cos (\alpha \pi / 2)}{\pi} \int_{-\infty}^{+\infty}|x|^{\alpha}(A(t)+i x)^{-1} u d x
$$

for all $u$ in $D(A(t))$ and $0<\alpha<1$.

4. Differentiability of square root. In this section, we will use the representation (12) in order to study the differentiability of $A(t)^{1 / 2}$ in the sense of the next definition.

DEFINITION 1. Let $\{A(t)$, t real $\}$, be a family of operators with constant domain $D=D(A(t))$ and range in a Banach space $B$. We say that $A(t)$ is strongly continuously differentiable with respect to $t$, when for all $u$ in $D$, the vector $v(t)=A(t) u$ is continuously differentiable with respect to the norm of $B$, that is,

$$
\lim _{t \rightarrow t_{0}: t \neq t_{0}}\left(t-t_{0}\right)^{-1}\left(v(t)-v\left(t_{0}\right)\right)
$$

exists in the sense of the norm of $B$ and is continuous for all $u$ in $D$ and all $t_{0}$.

When $A(t)$ is differentiable, the limit (13) is called the derivative of $A(t)$ and is represented by

$$
\left.\frac{d A(t)}{d t}\right|_{t=t_{0}}, \quad \frac{d A(t)}{d t} \text { or } A^{\prime}(t) .
$$

Suppose now that $\{A(t), t \geqq 0\}$, is a family of operators in a Hilbert space $H$, as in $\$ 2$ and satisfying the Assumptions (I), (II). In the following, we will prove that if 
$A(t)$ is strongly continuously differentiable and the commutator $C(t)$ of $A(t)$ and $A^{\prime}(t)$ satisfies the conditions $\gamma_{1}, \gamma_{2}$ below, then it follows that $A(t)^{1 / 2}$ is differentiable in the same sense.

By hypotheses

$$
\lim _{t \rightarrow t_{0}}\left(t-t_{0}\right)^{-1}\left(A(t) \omega-A\left(t_{0}\right) \omega\right)
$$

exist for all $\omega$ in $D=D(A(t))$. If $u \in H, \omega_{t_{0}}(x)=\left(A\left(t_{0}\right)+i x\right)^{-1} u$ belongs to $D$. Hence, it follows

$$
\lim _{t \rightarrow t_{0}}\left(t-t_{0}\right)^{-1}\left(A(t)-A\left(t_{0}\right)\right)\left(A\left(t_{0}\right)+i x\right)^{-1} u
$$

exists for all $u$ in $H$ and all real $x$. Since the bounded operators $(A(t)+i x)^{-1}$ are continuous in $t$, it follows that the following limit exists:

$$
\lim _{t \rightarrow t_{0}}\left(t-t_{0}\right)^{-1}\left[(A(t)+i x)^{-1}\left[A(t)-A\left(t_{0}\right)\right]\left(A\left(t_{0}\right)-i x\right)^{-1}\right] u .
$$

It follows that

$$
\lim _{t \rightarrow t_{0}}\left(t-t_{0}\right)^{-1}\left[(A(t)-i x)^{-1}-\left(A\left(t_{0}\right)-i x\right)^{-1}\right] u
$$

exists for all $u$ in $H$, because

$$
(A(t)+i x)^{-1}-\left(A\left(t_{0}\right)+i x\right)=-(A(t)+i x)^{-1}\left[A(t)-A\left(t_{0}\right)\right]\left(A\left(t_{0}\right)+i x\right)^{-1} .
$$

Hence, if $A(t)$ is strongly continuously differentiable, it follows that $(A(t)+i x)^{-1}$ is differentiable in the same sense and by (14) we have

$$
\frac{\partial}{\partial t}(A(t)+i x)^{-1}=(A(t)+i x)^{-1} \frac{d A(t)}{d t}(A(t)+i x)^{-1} .
$$

Assuming certain hypotheses on the commutator $C(t)$ of $A(t)$ and $A^{\prime}(t)$, we shall prove the convergence of the integral

$$
\int_{0}^{\infty} x^{1 / 2}(A(t)+i x)^{-1} A^{\prime}(t)(A(t)+i x)^{-1} u d x
$$

for all $u$ in $D\left(A(t)^{1 / 2}\right)$.

Let $A=A\left(t_{0}\right)$ be a fixed operator of the family $\{A(t), t \geqq 0\}$. Since $D\left(A^{2}\right) \subseteq D(A)$, we have, for all $u \in D\left(A^{2}\right), k\|u\| \leqq(A u \mid u) \leqq\|A u\|$ and

$$
\left(A^{2} u \mid u\right)=(A u \mid A u)=\|A u\|^{2} \geqq k^{2}(u \mid u)
$$

where $k=k\left(t_{0}\right)$, which proves that $A^{2}$ is bounded below, with lower bound $k^{2}$.

By the same method used in $\S 2$, to construct $D\left(A^{1 / 2}\right)$ from $D(A)$, we can construct $D(A)$ from $D\left(A^{2}\right)$. Furthermore, we know when $D\left(A(t)^{2}\right)=$ constant implies $D(A(t))=$ constant. Consequently, let $A(t)^{2}, A(t), A(t)^{1 / 2}$ have constant domains 
and let $A(t)$ be strongly continuously differentiable. Let us suppose that the commutator $C(t)$ of $A(t)$ and $A^{\prime}(t)$ satisfies the following conditions:

$\left(\gamma_{1}\right) D\left(A(t)^{2}\right) \subseteq D(C(t))$

$\left(\gamma_{2}\right)$ There exists $\gamma, 0 \leqq \gamma<1$ and a bounded positive continuous function $c(t)$, $t \geqq 0$, such that

$$
\|C(t) u\| \leqq c(t)\left\{\left\|A(t)^{2} u\right\|^{\gamma} \cdot\|u\|^{1-\gamma}+\|u\|\right\}
$$

for all $u$ in $D\left(A(t)^{2}\right)$.

We shall prove that with the hypotheses $\left(\gamma_{1}\right)$ and $\left(\gamma_{2}\right)$ on the commutator $C(t)$, the integral

$$
\int_{0}^{\infty} x^{1 / 2}(A(t)+i x)^{-1} A^{\prime}(t)(A(t)+i x)^{-1} u d x
$$

is convergent for all $u$ in $D\left(A(t)^{1 / 2}\right)$ or equivalently

$$
I=\int_{0}^{\infty} x^{1 / 2}(A(t)+i x)^{-1} A^{\prime}(t)(A(t)+i x)^{-1} A(t)^{-1 / 2} u d x
$$

is convergent for all $u$ in $H$.

In order to make the calculations easier, we shall write $A=A(t), A^{\prime}=A^{\prime}(t)$ and $A(t)^{-1 / 2}=A^{-1 / 2}$. Hence, we have $A A^{\prime}-A^{\prime} A=C$ or

$$
(A+i x) A^{\prime}-A^{\prime}(A+i x)=C \text {. }
$$

Multiplying on both sides by $(A+i x)^{-1}$, we obtain

$$
A^{\prime}(A+i x)^{-1}-(A+i x)^{-1} A^{\prime}=(A+i x)^{-1} C(A+i x)^{-1}
$$

or

$$
(A+i x)^{-1} A^{\prime}=A^{\prime}(A+i x)^{-1}-(A+i x)^{-1} C(A+i x)^{-1} .
$$

Substituting the last expression in the integral $I$, we obtain:

$$
I=\int_{0}^{\infty} x^{1 / 2} A^{\prime}(A+i x)^{-2} A^{-1 / 2} u-x^{1 / 2}(A+i x)^{-1} C(A+i x)^{-2} A^{-1 / 2} u d x
$$

We have

$$
\begin{aligned}
I_{1} & =\int_{0}^{\infty} x^{1 / 2} A^{\prime}(A+i x)^{-2} A^{-1 / 2} d x \\
& =A^{\prime} \int_{0}^{\infty} x^{1 / 2}\left(\int_{\mu \geqq \mu_{0}>0}^{\infty}(\mu+i x)^{-2} \mu^{-1 / 2} d E(\mu)\right) d x \\
& \left.=A^{\prime} \int_{\mu \geqq \mu_{0}>0}^{\infty} \mu^{-1 / 2}\left(\int_{0}^{\infty}(\mu+i x)^{-2} x^{1 / 2} d x\right) d E(\mu)\right) .
\end{aligned}
$$

We know that

$$
\int_{0}^{\infty} x^{1 / 2}(\mu+i x)^{-2} d x=\int_{0}^{\infty} \mu^{1 / 2} \eta^{1 / 2} \mu^{-2}(1+i \eta) \mu d \eta=h \mu^{-1 / 2}
$$

after the substitution, where $h$ is the value of the integral in $\eta$. 
Hence it follows

which is bounded.

$$
I_{1}=k A^{\prime} \int_{\mu \geqq \mu>0_{0}^{0}}^{\infty} \mu^{-1} d E(\mu)=k A^{\prime} A^{-1}
$$

Let $I_{2}$ be the integral

$$
I_{2}=\int_{0}^{\infty} x^{1 / 2}(A+i x)^{-1} C(A+i x)^{-2} A^{-1 / 2} u d x .
$$

To prove the convergence of $I_{2}$, we have by $\gamma_{1}, \gamma_{2}$ :

$$
\begin{aligned}
& \left\|x^{1 / 2}(A+i x)^{-1} C(A+i x)^{-2} A^{-1 / 2} u\right\| \\
& \quad \leqq x^{1 / 2}\left\|(A+i x)^{-1}\right\| \cdot\left\|C(A+i x)^{-2} A^{-1} u\right\| \\
& \quad \leqq k x^{1 / 2}\left\|(A+i x)^{-1}\right\|\left\{\left\|A^{2}(A+i x)^{-2} A^{-1 / 2} u\right\|^{\gamma} \cdot\left\|(A+i x)^{-2} A^{-1 / 2} u\right\|^{1-\gamma}\right. \\
& \left.+\left\|(A+i x)^{-2} A^{-1 / 2} u\right\|\right\} .
\end{aligned}
$$

Note that $k$ is the continuous function $c(t)$ of the hypotheses $\gamma_{2}$.

For large values of $x$, we have

$$
\begin{gathered}
\left\|(A+i x)^{-1}\right\| \leqq x^{-1}\left\|(A+i x)^{-2} A^{-1 / 2}\right\| \leqq K x^{-5 / 2} \\
\left\|A^{2}(A+i x)^{-2} A^{-1 / 2} u\right\| \leqq K x^{-1 / 2}\|u\| .
\end{gathered}
$$

Finally we have

$$
\begin{aligned}
& \left\|x^{1 / 2}(A+i x)^{-1} C(A+i x)^{-2} A^{-1 / 2} u\right\| \\
& \quad \leqq k x^{1 / 2} x^{-1}\left\{K^{\gamma} x^{-\gamma / 2}\|u\|^{\gamma} K^{1-\gamma} x^{-5(1-\gamma) / 2}\|u\|^{1-\gamma}+K x^{-5 / 2}\|u\|\right\} \\
& \quad=\left\{K_{1} x^{-5+\gamma}+K_{1} x^{-2}\right\}\|u\| .
\end{aligned}
$$

Hence it follows that the integral $I_{2}$ is convergent, because $0 \leqq \gamma<1$.

Whence, it follows that with the hypotheses $\left(\gamma_{1}\right),\left(\gamma_{2}\right)$ on the commutator, the integral

$$
\left(\pi 2^{1 / 2}\right)^{-1} \int_{-\infty}^{+\infty} x^{1 / 2} \frac{\partial}{\partial t}(A(t)+i x)^{-1} u d x
$$

converges and it is equal to the derivative of the integral, i.e., the vector $v(t)$ $=A(t)^{1 / 2} u$ is strongly continuously differentiable for all $u$ in $D\left(A(t)^{1 / 2}\right)$.

REMARK. The above proof is an adaptation for the present purpose of an argument given by F. Browder, Commutators with powers of an unbounded operator in Hilbert space, Proc. Amer. Math. Soc. 16 (1965), 1211-1213.

5. Cauchy problem for wave equations. Let $\{A(t), t \geqq 0\}$ be a family of operators as in $\S 2$. Hence by Theorem $1, \S 2$, it follows that $D\left(A(t)^{1 / 2}\right)$ is also independent of $t$. Let us represent by $C^{2}(R, H)$, the set of vector functions $u=u(t)$ from $R$ into $H$, which are twice strongly continuously differentiable with respect to $t$. Let $M(u)$ be a function, not necessarily linear from $D\left(A(0)^{1 / 2}\right)$ into $D\left(A(0)^{1 / 2}\right)$ satisfying the conditions (I), (II), (III), (IV) of Browder [1]. Let us suppose $D\left(A(0)^{1 / 2}\right)$ equipped with the norm $\|\quad\|_{w_{0}}$ defined by $\|u\|_{w_{0}}=\left\|A(0)^{1 / 2} u\right\|$. 
Definition 1. We call nonlinear wave equations in $H$, with time variable coefficients, all the equations of the form

$$
d^{2} u / d t^{2}+A(t) u+M(u)=0
$$

with $u \in C^{2}(R, H), A(t)$ and $M(u)$ as above.

We will consider the Cauchy problem

$$
\begin{gathered}
d^{2} u / d t^{2}+A(t) u+M(u)=0, \\
u(0)=\varphi, \quad u^{\prime}(0)=\psi,
\end{gathered}
$$

for $t \geqq 0, \varphi \in D(A(0))$ and $\psi \in D\left(A(0)^{1 / 2}\right)$.

Definition 2. A function $u$ in $C^{2}(\boldsymbol{R}, H)$, is said to be a strict solution of the Cauchy problem (16) and (17), when $u(t) \in D(A(0)), t \geqq 0, d u / d t \in D\left(A(0)^{1 / 2}\right)$, $A(t) u(t), d^{2} u / d t^{2}, A(t)^{1 / 2} u(t), A(t)^{1 / 2}(d u / d t)$ are uniformly continuous on every interval $[0, T], T>0$, and the system (16) and (17) is satisfied.

To prove the existence and uniqueness of strict solutions for (16) and (17), we need the following condition about $A(t)^{1 / 2}$.

Assumption III. The operator $A(t)^{1 / 2}$ is strongly continuously differentiable for all $t \geqq 0$ and $\left(d A(t)^{1 / 2} / d t\right) A(t)^{-1 / 2}$ is bounded by a bounded positive continuous function.

Now, we are in conditions to prove the main results in this paragraph, that is, the Theorems 1 and 2 below. The first one gives the existence of strict solutions and the second gives bounds for these solutions and their continuous dependence on the initial values.

TheOREM 1. Let $\{A(t), t \geqq 0\}$ be a family of operators in a Hilbert space $H$, satisfying the Assumptions I, II in $\S 2$, III in $\$ 4$, and $M(u)$ the mapping fixed before. Then, the system (16) and (17) has a strict solution $u=u(t), t \geqq 0$, provided $\varphi \in D(A(0)), \psi \in D\left(A(0)^{1 / 2}\right)$ and

$$
\|\varphi\|_{w_{0}}^{2}+\|\psi\|^{2}<C
$$

where $C$ is a positive constant.

THEOREM 2. Under the hypotheses of Theorem 1, for each $T>0$, if $u, u_{1}$ are solutions of (16) and (17) corresponding to the initial data $\varphi, \psi, \varphi_{1}, \psi_{1}$ satisfying the condition (18), we have

$$
\left\|A(t)^{1 / 2} u(t)-A(t)^{1 / 2} u_{1}(t)\right\|^{2}+\left\|u^{\prime}(t)-u_{i}^{\prime}(t)\right\|^{2} \leqq k(T, C)\left\{\left\|\varphi-\varphi_{1}\right\|_{w_{0}}^{2}+\left\|\psi-\psi_{1}\right\|^{2}\right\}
$$

for all $t$ in $[0, T]$.

THEOREM 3. If we assume the hypotheses of Theorem 1 and hypotheses (a) and (b) of Lemma 4 and

$$
\|A(0) \varphi\|^{2}+\|\varphi\|_{w_{0}}^{2}+\|\psi\|^{2}<C^{1}\left\|A(0) \varphi_{1}\right\|^{2}+\left\|\varphi_{1}\right\|_{w_{0}}^{2}+\|\psi\|^{2}<C
$$


we have

$$
\begin{aligned}
\left\|u^{\prime}(t)-u_{i}^{\prime \prime}(t)\right\|^{2}+\left\|A(t)^{1 / 2} u^{\prime}(t)-A(t)^{1 / 2} u_{1}(t)\right\|^{2} & \\
& \leqq k(T, C)\left\{\left\|\varphi-\varphi_{1}\right\|_{w_{0}}^{2}+\left\|A(0) \varphi-A(0) \varphi_{1}\right\|^{2}+\left\|\psi-\psi_{1}\right\|^{2}\right\}
\end{aligned}
$$

for each pair of solutions $u, u_{1}$ of (16) and (17) corresponding to $[\varphi, \psi],\left[\varphi_{1}, \psi_{1}\right]$.

COROLlaRY. The solution of the system (16) and (17) with conditions (19), is uniquely determined by the initial data $\varphi, \psi$.

The proofs of the Theorems 1, 2 and 3, follow from the lemmas below. First of all, we shall do a change of variable in order to transform the system (16) and (17) in an equivalent first order system in a convenient Hilbert space $\mathscr{H}$.

If we take $v=d u / d t$ in (16) and (17), we obtain

$$
\begin{gathered}
d u / d t=v, \quad d v / d t=-A(t) u-M(u), \\
u(0)=\varphi, \quad v(0)=\psi .
\end{gathered}
$$

If we take $x=A(t)^{1 / 2} u, y=v$ in (16) and (17) and use the Assumption III, we obtain

$$
\begin{gathered}
d x / d t=\frac{d A(t)^{1 / 2}}{d t} A(t)^{-1 / 2} x+A(t)^{1 / 2} y, \\
d y / d t=-A(t)^{1 / 2} x-M\left(A(t)^{-1 / 2} x\right) ; \\
x(0)=A(0)^{1 / 2} \varphi, \quad y(0)=\psi .
\end{gathered}
$$

Let us consider the Hilbert space $\mathscr{H}=H \oplus H$, direct sum of $H$ plus $H$ and we shall represent its elements by column vectors $\left(\begin{array}{l}x \\ y\end{array}\right)$ instead of pairs $(x, y)$ as is usually done. We have

$$
\left(\left(\begin{array}{l}
x \\
y
\end{array}\right) \mid\left(\begin{array}{l}
z \\
w
\end{array}\right)\right)=(x \mid z)+(y \mid w) \quad\left\|\left(\begin{array}{l}
x \\
y
\end{array}\right)\right\|^{2}=\|x\|^{2}+\|y\|^{2}
$$

for the inner product and norm in $\mathscr{H}$.

Hence, it follows that the Cauchy problem (16) and (17) in $H$, is equivalent to the following Cauchy problem in $\mathscr{H}$ :

$$
\begin{aligned}
d w / d t & =C(t) w+f(t, w), \\
w(0) & =\Phi,
\end{aligned}
$$

where

$$
\begin{aligned}
& w=\left(\begin{array}{l}
x \\
y
\end{array}\right), \\
& C(t)=\left(\begin{array}{cc}
0 & A(t)^{1 / 2} \\
-A(t)^{1 / 2} & 0
\end{array}\right), \quad \Phi=\left(\begin{array}{c}
A(0)^{1 / 2} \varphi \\
0
\end{array}\right), \\
& B(t)=\left(\begin{array}{ccc}
\frac{d A(t)^{1 / 2}}{d t} & A(t)^{-1 / 2} & 0 \\
0 & & 0
\end{array}\right) \text {, } \\
& g_{t}(w)=\left(\begin{array}{c}
0 \\
-M\left(A(t)^{-1 / 2} x\right)
\end{array}\right) \text { and } f(t, w)=B(t) w+g_{t}(w) \text {. }
\end{aligned}
$$


REMARK 1. The operator $B(t)$ is bounded by Assumption III. If $C>0$ is the constant in the condition (18), then $\|\Phi\|^{2}<C$. Furthermore, we observe that $0 \in D(C(0)), D(C(t))$ is independent of $t$ and dense in $\mathscr{H}$. The adjoint of $C(t)$ is $-C(t)$, which is the transpose of $C(t)$.

Kato in [6] has studied the Cauchy problem (24) and (25) in the linear case, that is to say, when $f(t, w)$ is a function $f(t)$ from $R$ into a Banach space $\mathscr{B}$, strongly continuous in $t$ on an interval $[a, b]$. He proves the existence and uniqueness for the strict solutions, i.e., the existence of a $w=w(t)$ which is strongly, continuously differentiable, where $C(t) w(t)$ is strongly continuous and $w=w(t)$ satisfies the system $w^{\prime}(t)=C(t) w(t)+f(t), w(0)=w_{0}$. We shall use Kato's results, when we apply the Picard-Banach fixed point theorem to the system (24) and (25), in a convenient metric space, which shall be defined later. First of all, we will establish the connection between Kato's results and the Cauchy problem (24) and (25), (cf. Lemma 1 below).

DEFINITION 3. By the transition mapping for the equation

$$
d w / d t=C(t) w, \quad 0 \leqq t \leqq T,
$$

we mean a family of operators $U(t, s), 0 \leqq s \leqq t \leqq T$ defined on $H$, such that $w(t)$ $=U(t, s) w_{0}$ is the solution of (26) with the initial condition $w(s)=w_{0}$, for $0 \leqq s \leqq t \leqq T$.

LEMMA 1. Let $C(t)$ be the operator in the equation (24). Then, for all real numbers $\lambda>0$, we have:

(a) The operator $\lambda I-C(t)$ has a bounded inverse $(\lambda I-C(t))^{-1}$ defined on $H$ and

for all $t$ in $[0, T]$.

$$
\left\|(\lambda I-C(t))^{-1}\right\|<\lambda^{-1}
$$

(b) The bounded operators $C(t)(\lambda I-C(0))^{-1}$ are strongly continuously differentiable for all $t$ in $[0, T]$.

(c) The equation (26), for $C(t)$ as in (28), has only one solution $w=w(t)$ in an interval $[0, T]$, such that $w(0)=w_{0}$ for a given $w_{0}$ in $D(C(t))$.

Proof of (a). Since $C(t)=-C(t)$, we have

$$
2 \operatorname{Re}(C(t) w \mid w)=(C(t) w \mid w)+(C(t) w \mid w)=0 .
$$

If $(\lambda I-C(t)) w=z$, taking the inner products of both sides and taking real parts, we obtain $\lambda\|w\| \leqq\|z\|$. Hence, it follows that if $z=0$, we have $w=0$, which implies that the kernel of $\lambda I-C(t)$ is $\{0\}$. Therefore, $(\lambda I-C(t))^{-1}$ exists for all $\lambda>0$ and we have $\left\|(\lambda I-C(t))^{-1}\right\|<\lambda^{-1}$. To complete the proof, we have to prove that the range of $\lambda I-C(t)$ is the whole of $\mathscr{H}$. Since $\lambda I-C(t)$ is closed and $(\lambda I-C(t))^{-1}$ is bounded on its domain, we have the range of $\lambda I-C(t)$ is closed in $\mathscr{H}$; therefore, it is sufficient to prove that it is dense in $\mathscr{H}$. Let $v$ in $\mathscr{H}$ be orthogonal to the range of $\lambda I-C(t)$. We have $((\lambda I-C(t)) u \mid v)=0$ for all $u$ in $D(\lambda I-C(t))$ which is dense in $\mathscr{H}$. It follows that $v \in D\left((\lambda I-C(t))^{*}\right)=D(\lambda I+C(t))$ and $(u \mid(\lambda I-C(t)) v)=0$ or $(\lambda I+C(t)) v=0$ which implies $v=0$. 
Proof of (b). By Assumption III, it follows that $C(t)$ is strongly continuously differentiable in $D$, for $t \in R$. Hence it follows that $C(t)(\lambda I-C(0))^{-1} u$ is continuously differentiable in $t$ for all $u$ in $\mathscr{H}$, since $(\lambda I-C(0))^{-1}$ is bounded.

Proof of (c). By parts (a), (b), it is permissible to construct the transition mappings $U(t, s)$, using Kato's method, associated to the operators $C(t)$ in an interval $[0, T]$. Hence, it follows that all solutions $w=w(t)$ of $(26)$, such that $w(0)=w_{0}$ belongs to $D$, are given by $w(t)=U(t, 0) w_{0}$ for $0 \leqq t \leqq T$. The uniqueness of the solution of (26), is still a consequence of Kato's results in [6].

LEMMA 2. The transition mappings for the equation (26) have norm one.

Proof. In fact, taking the inner product of both sides of the equation (26) with $w$, we obtain

$$
\left(\frac{d w}{d t} \mid w\right)=(C(t) w \mid w)
$$

Hence, taking the real parts, we have

$$
\frac{1}{2} \frac{d}{d t}\|w(t)\|^{2}=0 \text {. }
$$

Integrating the last equation between $s$ and $t$, it follows $\|w(t)\|=\|w(s)\|$ for all $0 \leqq s \leqq t \leqq T$. Since $w(t)=U(t, s) w_{s}$, for $w_{s}$ in $D$, is the solution of (26) such that $w(s)=w_{s}$. Whence, it follows

$$
\|U(t, s) w(s)\|=\|w(t)\|=\|w(s)\|
$$

for all $w(s)$ in $D$; therefore $\|U(t, s)\|=1$.

LEMMA 3. Let $u=u(t)$ be a solution of the system (16) and (17) on $[0, T]$, for a given $T>0$. Then we have

$$
\left\|A(t)^{1 / 2} u(t)\right\|^{2}+\left\|u^{\prime}(t)\right\|^{2} \leqq k(T, C)
$$

for all $t$ in $[0, T]$, provided $\|\varphi\|_{w_{0}}^{2}+\|\psi\|^{2} \leqq C$.

Proof. Let $u=u(t)$ be a solution of the system (16) and (17) on [0,T]. Then it follows that

$$
w(t)=\left(\begin{array}{c}
A(t)^{1 / 2} u \\
u^{\prime}
\end{array}\right)
$$

will be a solution of (24) and (25) on $[0, T]$, i.e., $w(t)$ satisfies the equation

$$
d w / d t=C(t) w+f(t, w), \quad 0 \leqq t \leqq T,
$$

with $C(t)$ and $f(t, w)$ defined in (24) and (25). Taking the inner product of both sides of the last equation with $w$ and taking real parts, we obtain

$$
\frac{1}{2} \frac{d}{d t}\|w(t)\|^{2}=\operatorname{Re}(f(t, w(t)) \mid w(t))
$$

since $\operatorname{Re}(C(t) w \mid w)=0$. Integrating the last scalar equation from 0 to $t \leqq T$, we have

$$
\|w(t)\|^{2}-\|w(0)\|^{2}=2 \operatorname{Re} \int_{0}^{t}(f(s, w(s)) \mid w(s)) d s .
$$


Since $f(t, w)=B(t) w+g(w)$, we obtain

$$
\begin{aligned}
\|w(t)\|^{2}-\|w(0)\|^{2}= & 2 \operatorname{Re} \int_{0}^{t}(B(s) w(s) \mid w(s)) d s \\
& -2 \operatorname{Re} \int_{0}^{t}\left(M(u(s)) \mid u^{\prime}(s)\right) d s
\end{aligned}
$$

By Browder's hypotheses on $M(u)$, we obtain

$$
\begin{aligned}
\|w(t)\|^{2}-\|w(0)\|^{2} \leqq & 2 \operatorname{Re} \int_{0}^{t}(B(s) w(s) \mid w(s)) d s \\
& +2 k_{0}\left(1+\int_{0}^{t}\left\|A(s)^{1 / 2} u(s)\right\|^{2} d s\right) .
\end{aligned}
$$

Since $2 \operatorname{Re}(B(s) w(s) \mid w(s)) \leqq b(s)\|w(s)\|^{2}$, with $b(s)$ continuous, we obtain

$$
\|w(t)\|^{2}-\|w(0)\|^{2} \leqq \int_{0}^{t} b(s)\|w(s)\|^{2} d s+2 k_{0}\left(1+\int_{0}^{t}\left\|A(s)^{1 / 2} u(s)\right\|^{2} d s\right) .
$$

If we take $g(t)=\|w(t)\|^{2}$, the last inequality can be written as

$$
g(t) \leqq k+k_{1} \int_{0}^{t} a(s) \cdot g(s) d s
$$

where $k=\|w(0)\|^{2}+2 k_{0}$. If we set

$$
a(t)=\int_{0}^{t} a(s) g(s) d s
$$

we have $z^{\prime}(t)=a(t) g(t)$ and then

$$
z^{\prime}(t) \leqq a(t)\left(k+k_{1} z(t)\right) .
$$

Hence it follows,

$$
\frac{d}{d t}\left(k+k_{1} z(t)\right) \leqq k_{1} a(t)\left(k+k_{1} z(t)\right)
$$

and integrating from 0 to $t \leqq T$, we obtain

$$
\left.\log \left(k+k_{1} z(s)\right)\right|_{0} ^{t} \leqq k_{1} \int_{0}^{t} a(s) d s
$$

or

$$
z(t) \leqq\left(k / k_{1}\right) \exp \left(k_{1} \int_{0}^{t} a(s) d s\right)-\left(k / k_{1}\right)
$$

Hence, it follows

$$
g(t) \leqq k \exp \left(k_{1} \int_{0}^{t} a(s) d s\right) \leqq k \exp (\lambda(T) T)
$$

where $\lambda(T)=k_{1} \max \{a(s): 0 \leqq s \leqq T\}$. Since $g(t)=\|w(t)\|^{2}=\left\|A(t)^{1 / 2} u(t)\right\|^{2}+\left\|u^{\prime}(t)\right\|^{2}$ and $k$ is fixed, the lemma is proved. 
LemMa 4. Let $u=u(t)$ be a solution of (16) and (17) on $[0, T]$. Suppose that $A(t)$ is twice strongly continuously differentiable with first and second derivatives satisfying the following conditions:

(a) $\left(A^{\prime}(t) u \mid u\right)<0$,

$$
\frac{d}{d t}\left(A^{\prime}(t) u \mid v\right) \leqq c(t)\left\{\left\|A(t)^{1 / 2} u\right\|^{2}+\left\|A(t)^{1 / 2} v\right\|^{2}\right\}
$$

(b) $\left|\left(A^{\prime \prime}(t) u \mid v\right)\right| \leqq k(t)\left\{\left\|A(t)^{1 / 2} u\right\|^{2}+\left\|A(t)^{1 / 2} v\right\|^{2}\right\}$,

with $c(t)$, and $k(t)$ positive continuous functions for $t \geqq 0$. Then, if $C>0$ is given, there exists $k(T, C)>0$ such that

$$
\left\|u^{\prime \prime}(t)\right\|^{2}+\left\|A(t)^{1 / 2} u^{\prime}(t)\right\|^{2} \leqq k(T, C)
$$

for all $t$ in $[0, T]$, provided

$$
\|A(0) \varphi\|^{2}+\|\varphi\|_{w_{0}}^{2}+\|\psi\|_{w_{0}}^{2}<C .
$$

Proof. By Lemma 3, there exists a constant $k_{C, T}>0$ such that

$$
\left\|u^{\prime}(t)\right\|^{2}+\left\|A(t)^{1 / 2} u(t)\right\|^{2} \leqq k_{C, T}
$$

for all $t$ in $[0, T]$. If we take $k_{C, T}$ as the constant $C$ in the Assumption III of Browder [4], we can obtain bounds for $\|(d / d t) M(u(t))\|$. Let $h$ be a positive real number, such that $0<t+h<T$. Then, $\delta_{h}(u)(t)=h^{-1}(u(t+h)-u(t))$, is a solution of the following equation:

$$
\begin{aligned}
\frac{d^{2}}{d t^{2}} \delta_{h}(u)(t)+A(t) \delta_{h}(u)(t) & +\frac{A(t+h) u(t+h)-A(t) u(t+h)}{h} \\
& +\frac{M(u(t+h))-M(u(t))}{h}=0 .
\end{aligned}
$$

By the hypotheses III in Browder [4], we have

$$
\left\|h^{-1}(M(u(t+h))-M(u(t)))\right\| \leqq k_{C, T}\left\|A(t)^{1 / 2} \delta_{h}(u)(t)\right\|+\varepsilon(h)
$$

where $\varepsilon(h) \rightarrow 0$ as $h \rightarrow 0$.

If we set $v=\delta_{h}(u)$, we have

$$
d^{2} / d t^{2}+A(t) v=g(t)
$$

with $g(t)=-A^{\prime}(\xi) u(t+h)+f(t)$, where $f(t)=-h^{-1}(M(u(t+h))-M(u(t)))$ and $\xi=t+h \Theta, 0<\Theta<1$. Hence it follows that

is a solution of

$$
w=\left(\begin{array}{c}
A(t)^{1 / 2} v \\
v^{\prime}
\end{array}\right)
$$

$$
d w / d t=C(t) w+g(t, w), \quad 0<t<T,
$$

in $\mathscr{H}$, with $g(t, w)=B(t) w+h(t)$, where $h(t)$ is the vector

$$
h(t)=\left(\begin{array}{c}
0 \\
-A^{\prime}(\xi) u(t+h)+f(t)
\end{array}\right) .
$$


Taking the inner product of both sides of the last equation with $w$ and taking the real parts, we obtain:

$$
(d / d t)\|w(t)\|^{2}=2 \operatorname{Re}(g(t, w) \mid w)
$$

To estimate $2 \operatorname{Re}(g(t, w) \mid w)$, we have

$$
\operatorname{Re}(g(t, w) \mid w)=\operatorname{Re}(B(t) w \mid w)+\operatorname{Re}(h(t) \mid w) .
$$

With $\operatorname{Re}(B(t) w \mid w) \leqq b(t)\|w\|^{2}$, where $b(t)$ is a continuous function on $\boldsymbol{R}^{+}$. Further we have

$$
\operatorname{Re}(h(t) \mid w)=-\operatorname{Re}\left(A^{\prime}(\xi) u(t+h) \mid v^{\prime}\right)+\operatorname{Re}\left(f(t) \mid v^{\prime}\right)
$$

with

$$
\operatorname{Re}\left(f(t) \mid v^{\prime}\right) \leqq\|f(t)\|\left\|v^{\prime}\right\| \leqq k_{C, T}^{\prime}\left\{\left\|A(t)^{1 / 2} v\right\|^{2}+\left\|v^{\prime}\right\|^{2}\right\}+\varepsilon(h) .
$$

To estimate $\operatorname{Re}\left(A^{\prime}(\xi) u(t+h) \mid v^{\prime}\right)$, let us consider first of all $(d / d t)\left(A^{\prime}(\xi) u(t+h) \mid v\right)$. In fact,

$(d / d t)\left(A^{\prime}(\xi) u(t+h) \mid v\right)=\left(A^{\prime \prime}(\xi) u(t+h) \mid v\right)+\left(A^{\prime}(\xi) u(t+h)^{\prime} \mid v\right)+\left(A^{\prime}(\xi) u(t+h) \mid v^{\prime}\right)$.

Then we have:

$$
\begin{aligned}
-\operatorname{Re}\left(A^{\prime}(\xi) u(t+h) \mid v^{\prime}\right)= & -\operatorname{Re}(d / d t)\left(A^{\prime}(\xi) u(t+h) \mid v\right) \\
& +\operatorname{Re}\left(A^{\prime \prime}(\xi) u(t+h) \mid v\right)+\operatorname{Re}\left(A^{\prime}(\xi) u^{\prime}(t+h) \mid v\right) .
\end{aligned}
$$

Therefore, for $h$ sufficiently small, we have, by hypotheses $\operatorname{Re}\left(A^{\prime}(\xi) u^{\prime}(t+h) \mid v\right) \leqq 0$. Still by the assumptions (a) and (b) we can estimate

$$
-\operatorname{Re}(d / d t)\left(A^{\prime}(\xi) u(t+h) \mid v\right) \text { and } \operatorname{Re}\left(A^{\prime \prime}(\xi) u(t+h) \mid v\right) .
$$

For that it is important to observe that $A^{1 / 2}(t) A^{-1 / 2}(s)$ is uniformly bounded on $[0, T]$. Furthermore, if $u$ is a solution of $u^{\prime \prime}+A(t) u+M(u)=0$, by Lemma 3 , we know that $A^{1 / 2}(t+h) u(t+h)$ is uniformly bounded on $[0, t]$ and then it follows that $A^{1 / 2}(t) u(t+h)$ is also uniformly bounded on $[0, T]$. After suitable computations, we have

$$
(d / d t)\|w(t)\|^{2} \leqq a+b\|w(t)\|^{2}
$$

where $a$ is a constant $\gamma(T, C)+\varepsilon(h)$ and $b=b(C, T)$, and both constants are positive.

If we set $z(t)=\|w(t)\|^{2}$ we obtain the differential inequality $z^{\prime}(t) \leqq a+b z(t)$, $0 \leqq t \leqq T$, which implies

$$
(d / d t)\left[e^{-b t} z(t)\right] \leqq a e^{-b t}
$$

Integrating the last inequality, we obtain

$$
z(t) \leqq e^{b t} z(0)+(a / b)\left(e^{b t}-1\right), \quad 0 \leqq t \leqq T .
$$

Substituting $z$ by $\|w(t)\|^{2}=\left\|A(t)^{1 / 2} v(t)\right\|^{2}+\left\|v^{\prime}(t)\right\|^{2}$ and $v(t)=h^{-1}(u(t+h)-u(t))$ in the last inequality and then making $h \rightarrow 0$, we obtain

$$
\left\|u^{\prime \prime}(t)\right\|^{2}+\left\|A(t)^{1 / 2} u^{\prime}(t)\right\|^{2} \leqq k^{\prime}(C, T)\|A(0) \varphi+M(\varphi)\|^{2}+\left\|A(0)^{1 / 2} \psi\right\|^{2}+k^{\prime \prime}(C, T) .
$$


By hypotheses, we can estimate $M(\varphi)$ and then we obtain

$$
\left\|u^{\prime \prime}(t)\right\|^{2}+\left\|A(t)^{1 / 2} u^{\prime}(t)\right\|^{2} \leqq k(T, C), \quad 0 \leqq t \leqq T .
$$

REMARK. The proof of Lemma 4 can be simplified if we take the inner product of $v^{\prime}$ with both sides of $v^{\prime \prime}+A(t) v+A^{\prime}(\xi) u(t+h)=f(t)$.

Proof of Theorem 2(a). Let $u=u(t), u_{1}=u_{1}(t)$ be two solutions of (16), (17) corresponding to the initial data $\varphi, \psi, \varphi_{1}, \psi_{1}$. If we set $w=u-u_{1}$, we have that $w$ is a solution of

$$
d^{2} w / d t^{2}+A(t) w+M(u)-M\left(u_{1}\right)=0
$$

with the initial conditions

$$
w(0)=\varphi-\varphi_{1}, \quad w^{\prime}(0)=\psi-\psi_{1} .
$$

Hence, it follows that

is a solution of

$$
v=\left(\begin{array}{c}
A(t)^{1 / 2} w \\
w^{\prime}
\end{array}\right)
$$

$$
d v / d t=C(t) v+f(t, v)
$$

with $f(t, v)=B(t) v+g(v)$ and

$$
g(v)=\left(\begin{array}{c}
0 \\
M\left(u_{1}(t)\right)-M(u(t))
\end{array}\right) .
$$

By Lemma 3 and Assumption (M1), we can estimate $g(v)$ and we obtain

$$
\|g(v)\| \leqq\left\|M(u(t))-M\left(u_{1}(t)\right)\right\| \leqq k_{c}\left\|A(t)^{1 / 2} v(t)\right\| .
$$

Taking the inner product of both sides of the equation (27), with $v$, and taking the real parts, we have

or

$$
(d / d t)\|v(t)\|^{2}=2 \operatorname{Re}(f(t, v(t)) \mid v(t))
$$

$$
(d / d t)\|v(t)\|^{2} \leqq c(t)\|v(t)\|^{2}
$$

where $c(t)$ is a real continuous function on $\boldsymbol{R}$. If we define $z(t)=\|v(t)\|^{2}$, we have $z^{\prime}(t) \leqq c(t) z(t)$.

Since $a(t) \neq 0, u$ and $u_{1}$ are not equal, we obtain from the last inequality $z(t)$ $\leqq z(0) k(C, T)$.

When we substitute $z(t)$ as a function of $u$ and $u_{1}$, the last inequality proves (a) of Theorem 2.

Proof of Theorem 2(b). If we set $w=u-u_{1}$, it follows that

satisfies the equation

$$
\delta_{h}(w)(t)=[w(t+h)-w(t)] h^{-1}
$$

$$
\begin{aligned}
\left(d^{2} / d t^{2}\right) & \delta_{h}(w)(t)+A(t) \delta_{h}(w)(t)+A^{\prime}(\xi) w(t+h) \\
& +h^{-1}(M(u(t+h))+M(u(t)))-h^{-1}\left(M\left(u_{1}(t+h)\right)-M\left(u_{1}(t)\right)\right)=0 .
\end{aligned}
$$


By the hypotheses on $u, u_{1}$, by Assumption IV in Browder [1], and certain calculations, we have

$$
\begin{aligned}
\| h^{-1}(M(u(t+h))-M(u(t))) & -h^{-1}\left(M\left(u_{1}(t+h)\right)-M\left(u_{1}(t)\right)\right) \| \\
& \leqq k_{C, T}^{\prime}\left\{\left\|A(t)^{1 / 2} \delta_{h}(w)(t)\right\|+\left\|A(t)^{1 / 2} w(t)\right\|\right\}+\varepsilon(h) .
\end{aligned}
$$

It follows that if we take $v=\delta_{h}(w)$, the equation (28) can be written as

$$
d^{2} v / d t^{2}+A(t) v+A^{\prime}(\xi) w(t+h)+f(t)=0
$$

with

$$
\|f(t)\| \leqq k_{C, T}^{\prime}\left\{\left\|A(t)^{1 / 2} v(t)\right\|+\left\|A(t)^{1 / 2} w(t)\right\|\right\}+\varepsilon(h) .
$$

Taking the change of variables

$$
W=\left(\begin{array}{c}
A(t)^{1 / 2} v \\
v^{\prime}
\end{array}\right)
$$

as in the Lemma 4, the equation (29) can be written as

$$
(d / d t) W(t)=C(t) W(t)+g(t, W(t))
$$

with the same notation as in Lemma 4. Taking the inner product of both sides of the equation (30) with $W$ and taking real parts, we obtain:

We have

$$
(d / d t)\|W(t)\|^{2}=2 \operatorname{Re}(g(t, W(t)) \mid W(t)) .
$$

$$
\operatorname{Re}(g(t, W(t)) \mid W(t))=\operatorname{Re}(B(t) W(t) \mid W(t))
$$

We have

$$
-\operatorname{Re}\left(A^{\prime}(\xi) w(t+h) \mid v^{\prime}(t)\right)+\operatorname{Re}(f(t) \mid W(t)) .
$$

$$
\operatorname{Re}(B(t) W(t) \mid W(t)) \leqq b(t)\|W(t)\|^{2}
$$

with $b(t)$ continuous on $\boldsymbol{R}^{+}$.

$$
\operatorname{Re}(f(t) \mid W(t)) \leqq\left(1+5 k_{C, T}^{\prime 2}\right)\|W(t)\|^{2}+4 k_{C, T}^{\prime 2}\left\|A(t)^{1 / 2} w(t)\right\|+\varepsilon(h)
$$

and

$$
-\operatorname{Re}\left(A^{\prime}(\xi) w(t+h) \mid v^{\prime}\right) \leqq\left(2 h^{2} k_{1}(t)+1\right)\|w(t)\|^{2}+2 k_{1}(t)\left\|A(t)^{1 / 2} w(t)\right\|^{2} .
$$

Since $w(t+h)=h v+w$, it follows that

$$
\left\|A(t)^{1 / 2} w(t+h)\right\|^{2} \leqq 2 h^{2}\left\|A(t)^{1 / 2} v\right\|^{2}+2\left\|A(t)^{1 / 2} w(t)\right\|^{2} .
$$

Consequently we can say that

$$
(d / d t)\|W(t)\|^{2} \leqq \dot{K}^{\prime}(C, T, h)\left\{\|W(t)\|^{2}+\left\|A(t)^{1 / 2} w(t)\right\|^{2}+\left\|W^{\prime}(t)\right\|^{2}\right\}+\varepsilon(h)
$$

such that $\lim _{h \rightarrow 0} K^{\prime}(C, T, h)$ exists and is a constant which depends only on $C$ and $T$. If we set

we have

$$
a(t)=\|W(t)\|^{2}+\left\|A(t)^{1 / 2} w(t)\right\|^{2}+\left\|W^{\prime}(t)\right\|^{2}
$$

$$
z^{\prime}(t) \leqq a z(t)+\varepsilon(h) .
$$


Integrating the last inequality, we obtain

$$
z(t) \leqq K(C, T)(z(0)+\varepsilon(h)) .
$$

Substituting $z(t)$ in functions of $u$ and $u_{1}$, we obtain an inequality which implies that in Theorem 2.

Lemma 5. To prove Theorem 1 , it is sufficient to prove that for each $C>0$, there exists an interval $\left[0, T_{C}\right]$ such that the system (16) and (17) has a solution $u=u(t)$ on $\left[0, T_{C}\right]$ for all sets of data $[\varphi, \psi]$ such that

$$
\|A(0) \varphi\|^{2}+\left\|A(0)^{1 / 2} \psi\right\|^{2}+\left\|A(0)^{1 / 2} \varphi\right\|^{2}<C .
$$

Proof (Browder [1]). Suppose Theorem 1 is false. Hence it follows that for some $\varphi \in D(A(0)), \psi \in D\left(A(0)^{1 / 2}\right)$, there exists $T>0$ such that no solutions of (16) and (17) can be found outside the interval $[0, T]$. When $\varphi, \psi, C$ vary as in the hypotheses of Lemma 5 , we can construct (by the uniqueness given by the corollary of Theorem 2), a solution $u=u(t)$ of (16) and (17) defined on an interval $\left[0, T_{0}\right]$ with $T_{0} \leqq T$, such that $u=u(t)$ cannot be continued as a solution of (16) and (17) after $T_{0}$. By Lemmas 3 and 4 and by the Assumption I in Browder [1], we can obtain a constant $C_{1}$ given by

$$
C_{1}=\sup _{0 \leqq t \leqq T}\left\{\|A(t) u(t)\|^{2}+\left\|A(t)^{1 / 2} u^{\prime}(t)\right\|+\left\|A(t)^{1 / 2} u(t)\right\|^{2}\right\} .
$$

Let $T_{2}$ be any point inside the interval $\left[0, T_{0}\right]$ and let $\varphi_{1}, \psi_{1}$ be defined by $\varphi_{1}=u\left(T_{2}\right)$, $\psi_{1}=u^{\prime}\left(T_{2}\right)$. By the definition of $C_{1}$, we have

$$
\left\|A(0) \varphi_{1}\right\|^{2}+\left\|A(0)^{1 / 2} \psi_{1}\right\|^{2}+\left\|A(0)^{1 / 2} \varphi_{1}\right\|^{2} \leqq C_{1} .
$$

Hence, it follows that there exists a number $T_{1}=T_{C_{1}}>0$ such that the Cauchy problem (16) and (17) at the point $T_{2}$ has a solution $u_{1}=u_{1}(t)$ defined on the interval $\left[T_{2}, T_{2}+T_{1}\right]$ for all $T_{2}$ inside $\left[0, T_{0}\right]$. When $T_{2}=T_{0}-(1 / 2) T_{1}$, we obtain that $u_{1}=u_{1}(t)$ at $T_{2}$. By the uniqueness, $u$ and $u_{1}$ are equal on $\left[0, T_{0}\right]$ and $u=u(t)$ is continuable outside $\left[0, T_{0}\right]$ as a solution of (16) and (17) and we have a contradiction.

Now, we shall prove that for each $C>0$, there exists a $T_{0}>0$, depending only upon $C$ and not upon $\varphi \in D(A(0)), \psi \in D\left(A(0)^{1 / 2}\right)$, such that the system (16) and (17) has a strict solution on $\left[0, T_{C}\right]$, for each set of data $\varphi, \psi$, with

$$
\|A(0) \varphi\|^{2}+\|\psi\|_{W_{0}}^{2}+\|\varphi\|_{W_{0}}^{2}<C .
$$

Let us rewrite the system (16) and (17):

$$
\begin{gathered}
d^{2} u / d t^{2}+A(t) u+M(u)=0, \\
u(0)=\varphi, \quad u^{\prime}(0)=\psi .
\end{gathered}
$$

The system (31) and (32) is equivalent to the system

$$
\begin{aligned}
(d w / d t) & =C(t) w+f(t, w), \quad 0 \leqq t \leqq T, \\
w(0) & =\Phi,
\end{aligned}
$$


in the space $\mathscr{H}$, as in the system (24) and (25). By Kato [6] it follows that if $w=w(t)$ is a strict solution of (33) and (34) on $[0, T]$, then $w=w(t)$ is a solution of the following integral equation:

$$
w(t)=U(t, 0) \Phi+\int_{0}^{t} U(t, s) f(s, w(s)) d s .
$$

In fact, by Kato [6], for all $w_{0} \in D(C(0))$, we have

$$
(d / d t) U(t, s) w_{0}=-U(t, s) C(s) w_{0} .
$$

Hence, if $w=w(t), 0<t<T$ is a solution of (33) and (34), we have

or

$$
\frac{d}{d s} U(t, s) w(s)=-U(t, s) C(s) w(s)+U(t, s) \frac{d w(s)}{d s}
$$

$$
(d / d s) U(t, s) w(s)=U(t, s) f(s, w(s)) .
$$

Integrating (36) from 0 to $t<T$, we obtain the integral equation (35).

Since $f(t, w)$ belongs to $D(C(0))$, we can use Kato's results in [6], and conclude that $w(t)$ given by (33) is a strict solution of (33) and (34).

In order to complete the proof of Theorem 1, we have to prove that (35) has only one continuous solution on an interval $[0, T]$, for any 0 in $D$, with $\|\Phi\| \leqq C$, for a given $C>0$. For that, we will construct a mapping $S: X \rightarrow X$, where $X$ is a metric space which shall be defined later, such that for some sphere $X_{1} \subseteq X$ depending upon $C$ only, we have $S X_{1} \subseteq X$ and on $X_{1}$ the mapping $S$ is a contraction and its fixed point is equal to the solution of (35).

In fact, let $\Phi \in D$ be such that $\|\Phi\| \leqq C$, and $T>0$ is a real number which shall be chosen later, depending upon $C$. Let us consider the set $X=X(\Phi, C, T)$ of all the mappings $w:[0, T] \rightarrow \mathscr{H}$, such that $w=w(t)$ is strongly continuous for all $t$ on $[0, T]$ and $w(0)=0$. If we define in $X$ the distance between $w_{1}, w_{2}$ by

where

$$
d\left(w_{1}, w_{2}\right)=\left\|w_{1}-w_{2}\right\|_{X}
$$

$$
\|w\|_{X}=\sup _{0<t<T}\|w(t)\| .
$$

It follows that $X$ equipped with (37) is a complete metric space.

Now, suppose $S$ is defined on $X$ in the following way: for each $v$ in $X, w=S v$ is the function $w=w(t)$ defined on $[0, T]$ by

$$
w(t)=U(t, 0) \Phi+\int_{0}^{t} U(t, s)[B(s) v(s)+g(v(s))] d s
$$

where $U(t, s)$ is the transition mapping associated to $w^{\prime}(t)=C(t) w, B(t)$ and $g(v)$ are defined as in the system (24) and (25). Observe that the fixed points of (38) are the solutions of (35).

By Kato's results, $w(t)$ defined by (38) belongs to $X$ and thus, $S$ is a well defined mapping from $X$ into $X$. 
Now we shall prove that given $C>0$, such that $\|\Phi\|_{1}<C$, we have a positive number $C_{1}$, depending only upon $C$, such that we can fix a number $T>0$, depending only upon $C_{1}$ and therefore only on $C$, and such that if we consider the sphere

$$
X_{1}=\left\{v \in X \mid\|v\|_{X}<C_{1}\right\}
$$

in $X$, we have $S X_{1} \subseteq X_{1}$ and on $X_{1}$ the mapping $S$ is a contraction.

In fact, let $v \in X_{1}$ and $w=S v$. Hence we have by definition of $S$

$$
w(t)=U(t, 0) \Phi+\int_{0}^{t} U(t, s)[B(s) v(s)+g(v(s))] d s, \quad 0 \leqq t \leqq T
$$

whence it follows

$$
\|w(t)\| \leqq\|\Phi\|+\int_{0}^{t}\|B(s) v(s)+g(v(s))\| d s .
$$

REMARK 4. We know that the bounded operator $B(s)$ is continuous for $s \geqq 0$, and

$$
\|g(v(s))\|=\left\|M\left(A(t)^{-1 / 2} x\right)\right\| \leqq k(t)\|x\| \leqq k(t)\|v\|_{X} .
$$

By (39) we have

$$
\|w(t)\| \leqq\|\Phi\|+T\|v\|_{X} \sup _{0 \leqq t \leqq T} k^{\prime}(t)
$$

$k^{\prime}(t)$ continuous. Since $\|\Phi\|<C$, we take $C_{1}>2 C$ and since $k^{\prime}(t)$ is continuous, we chose $T>0$ such that

$$
\mathrm{T} \sup _{0 \leqq t \leqq T} k^{\prime}(t)<1 / 2
$$

which depends only upon $C$. It follows that if $v \in X_{1}, C_{1}$ picked out before, we have $S v \in X_{1}$ and then $S X_{1} \subseteq X_{1}$.

Let us prove now, that $S$ is a contraction. Suppose $v_{1}, v_{2}$ in $X_{1}$ and let $w_{1}, w_{2}$ be such that $S v_{1}=w_{1}$ and $S v_{2}=w_{2}$. Hence it follows

$$
\left(w_{1}-w_{2}\right)(t)=\int_{0}^{t} U(t, s)\left[B(s)\left(v_{1}-v_{2}\right)(s)+g\left(v_{1}(s)\right)-g\left(v_{2}(s)\right)\right] d s
$$

whence it follows

$$
\left\|w_{1}(t)-w_{2}(t)\right\| \leqq T\left\|v_{1}-v_{2}\right\|_{X} \sup _{0 \leqq t \leqq T} k^{\prime}(t)
$$

and taking $T$ as fixed before, we obtain

$$
\left\|S V_{1}-S V_{2}\right\|_{X} \leqq(1 / 2)\left\|V_{1}-V_{2}\right\|_{X}
$$

which proves that $S$ is a contraction. Q.E.D.

Applications. Let $G$ be a bounded domain in the real Euclidean space $\boldsymbol{R}^{N}$ of dimension $N$, with a smooth boundary $\partial G$. Let us suppose the Hilbert space $H$ equal to $L^{2}(G)$ of the classes of Lebesgue square-integrable functions with its usual inner product. 
We will consider a selfadjoint realization in $L^{2}(G)$ of a formal partial differential operator of second order, with coefficients depending on $x=\left(x_{1}, x_{2}, \ldots, x_{N}\right) \in G$ and on a positive real parameter $t$, the time variable. Thus, let $A(t)$ be the formal differential operator

$$
A(t)=-\sum_{i, k=1}^{N} \frac{\partial}{\partial x_{i}}\left[a_{i k}(x, t) \frac{\partial}{\partial x_{j}}\right]+c(x, t)
$$

where $a_{i j}(x, t), c(x, t)$ are real functions defined on $\partial \bar{G} \times \boldsymbol{R}$, for $i, k=1,2, \ldots, N$, $a_{i k}(x, t)=a_{k i}(x, t)$ for $1 \leqq i, k \leqq N$, and there exist two bounded positive continuous functions $C_{0}(t)$ and $C_{1}(t)$ such that

$$
C_{1}(t)|\xi|^{2} \geqq \sum_{i, k=1}^{N} a_{i k}(x, t) \xi_{i} \xi_{k} \geqq C_{0}(t)|\xi|^{2}
$$

for all $\xi=\left(\xi_{1}, \xi_{2}, \ldots, \xi_{N}\right)$ and $x=\left(x_{1}, x_{2}, \ldots, x_{N}\right) \in \bar{G}$ where $|\xi|^{2}=\xi_{1}^{2}+\xi_{2}^{2}+$ $\cdots+\xi_{N}^{2}$.

We suppose that $a_{i k}(x, t), C(x, t)$ are $C^{\infty}$ functions on $G \times \boldsymbol{R}$ with derivatives up to the order four bounded by a positive continuous function $\lambda(t)$ on $\bar{G} \times \boldsymbol{R}$. Further, we suppose that $a_{i k}(x, t), c(x, t)$ and $\partial a_{i j} / \partial x_{i}$, satisfy the Lipschitz condition with respect to $t$ uniformly in $x$ and that $\inf _{x} c(x, t)>0$ for all $t>0$.

Let $W^{m, p}(G)$ be the appropriate Sobolev space. We represent by $A_{2}(t)$ the realization of the formal differential operator $A(t)$ given by (40), under Dirichlet null boundary conditions, i.e., the domain of $A_{2}(t)$ is the set of functions $u$ in $W^{2,2}(G)$ such that $D^{\alpha} u_{\partial G}=0$ for all $|\alpha|<2$. It follows that $D\left(A_{2}(t)\right)$ is independent of $t$ and dense in $L^{2}(G)$. Further, $A_{2}(t)$ is a mapping from $L^{2}(G)$ into $L^{2}(G)$ which is selfadjoint and semibounded by a positive continuous function. We have, moreover, that $A_{2}(t)$ satisfies all the conditions assumed in Theorem 1. We check only the conditions $\gamma_{1}$ and $\gamma_{2}$ on the computator, (cf. $\S 3$ ). In fact, $D\left(A_{2}(t)^{2}\right)$ is the set of $u \in D\left(A_{2}(t)\right)$ such that $A_{2}(t) u \in D\left(A_{2}(t)\right)$. Thus, by regularity theory (cf. Browder [2, p. 52, Theorem 4]) it follows that $u \in W^{4,2}(G)$ and $\gamma_{1}$ is fulfilled.

Let $C_{2}(t)$ the commutator of $A_{2}(t)$ and $A_{2}^{\prime}(t)$, the derivative of $A_{2}(t)$, which has order three. For all $u \in D\left(C_{2}(t)\right)$ we have

$$
\left\|C_{2}(t) u\right\|_{0,2} \leqq \beta(t)\|u\|_{3,2}
$$

where $\beta(t)>0$ is a continuous function. By Browder [2, p. 42, Lemma 1], we have

$$
\|u\|_{3,2} \leqq\|u\|_{4,2}^{3 / 4} \cdot\|u\|_{0,2}^{1 / 4}
$$

for all $u \in D\left(A_{2}(t)^{2}\right)$. Again by Browder [2, p. 44], it follows that

$$
\|u\|_{4,2}^{3 / 4} \leqq k_{2}(t)\left\{\left\|A_{2}(t)^{2} u\right\|_{0,2}^{3 / 4}+\|u\|_{0,2}^{3 / 4}\right\} \text {. }
$$

Whence from (42) and (43) we have

$$
\|u\|_{3,2} \leqq k(t)\left\{\left\|A_{2}(t)^{2} u\right\|_{0,2}^{3 / 4} \cdot\|u\|_{0,2}^{1 / 4}+\|u\|_{0,2}\right\}
$$

for all $u$ in $D\left(A(t)^{2}\right)$. 
Finally by (42) and (45) we have

$$
\|C(t) u\|_{0,2} \leqq h(t)\left\{\left\|A_{2}(t)^{2} u\right\|_{0,2}^{3 / 4}\|u\|_{0,2}^{1 / 4}+\|u\|_{0,2}\right\}
$$

which is the condition $\left(\gamma_{2}\right)$ with $\gamma=3 / 4$ and this implies that $A(t)^{1 / 2}$ is continuously strongly differentiable.

Since $D^{\alpha}$, for $|\alpha| \leqq m$, are bounded mappings from $W^{m . p}(G)$ into $L^{p}(G)$, it follows that $d A_{2}(t)^{1 / 2} / d t$ is a bounded mapping from $D\left(A_{2}(t)^{2}\right)$ into $L^{2}(G)$. Whence it follows that $\left(d A_{2}(t)^{1 / 2} / d t\right) A(t)^{-1 / 2}$ is a bounded operator from $L^{2}(G)$ into $L^{2}(G)$.

\section{REFERENCES}

1. F. E. Browder, On nonlinear wave equations, Math. Z. 80 (1962), 249-264.

2. - On spectral theory of elliptic differential operators. I, Math. Ann. 142 (1961), 22-130.

3. F. E. Browder and W. A. Strauss, Scattering for nonlinear wave equations, Pacific J. Math. 13 (1963), 23-43.

4. F. E. Browder, Nonlinear equations of evolution, Ann. of Math. (2) 80 (1964), 485-523.

5. E. Heinz, Beiträge zur Störungstheorie der Spektralzerlegung, Math. Ann. 123 (1951), 415-438.

6. T. Kato, Integration of the equation of evolution in a Banach space, J. Math. Soc. Japan 5 (1953), 208-234.

7. - , Notes on some inequalities for nonlinear operators, Math. Ann. 125 (1952), 208-212.

8. J. L. Lions, Equations différentielles opérationnelles et problèmes aux limites, Springer, Berlin, 1961.

9. - Lectures on elliptic partial differential equations, Tata Institute Lecture Notes, Bombay, 1957.

10. F. Riesz and B. Sz. Nagy, Functional analysis, Ungar, New York, 1955.

11. I. Segal, Nonlinear semigroups, Ann. of Math. (2) 78 (1963), 339-364.

Instituto de Matemática Pura e Aplicada, Rio de JANEIRO, BRASIL

Faculdade de Filosofia da Universidade Federal do Rio de Janeiro, Rio de Janeiro, Brasil 